\title{
Reduction of Internal Standard Signals in Quantitative MALDI-TOF Mass Spectrometry
}

\author{
Walter B. Wilson ${ }^{1}$, Dickson M. Wambua ${ }^{1}$, Norman H. L. Chiu ${ }^{1,2^{*}}$ \\ ${ }^{1}$ Department of Chemistry and Biochemistry, University of North Carolina at Greensboro, Greensboro, USA; ${ }^{2}$ Department of \\ Nanoscience, Joint School of Nanoscience and Nanoengineering, Greensboro, USA. \\ Email: " prof.chiu@gmail.com
}

Received July $21^{\text {st }}, 2012$; revised August $24^{\text {th }}, 2012$; accepted September $1^{\text {st }}, 2012$

\begin{abstract}
The advantages of combining qualitative and quantitative analysis on a single analytical technique have further extended the applications of matrix-assisted laser desorption/ionization time-of-flight mass spectrometry (MALDI-TOF MS) to the quantitation of various biomolecules. To achieve absolute quantitation, it is necessary to perform a calibration with standard dilutions. For the purpose of measuring DNA samples, a pure DNA oligonucleotide at different concentrations was chosen as a standard to perform the calibration of Quantitative MALDI-TOF MS. In order to overcome the variation of signal intensity from repeated measurements of each DNA standard dilution, fixed amount of an internal standard was added into each DNA standard dilution. Instead of maintaining at a constant level, the signals of fixed amount of internal standard were decreased $73 \%$ from its initial level while the signals of DNA standard continued to increase within a linear dynamic range for quantitation from $0.20 \mu \mathrm{M}$ to $12.5 \mu \mathrm{M}$ of DNA. Attempts to identify the cause of signal reduction were systematically carried out. This is the first report on the extent of signal reduction in quantitative MALDI-TOF MS. These results represent a limitation on using MALDI-TOF MS to monitor the changes in concentration of two different compounds within a chemical or biological system.
\end{abstract}

Keywords: MALDI; Mass Spectrometry; Quantitation

\section{Introduction}

With the launch of commercialized MALDI-TOF MS instruments, the number of applications for MALDI-TOF MS technique has exponentially increased in the recent years [1-7]. MALDI-TOF has been widely used for the characterization of different biomolecules such as nucleic acids and proteins. The advantages of using MALDITOF MS include 1) $\leq 0.3 \mu \mathrm{L}$ of sample is required, 2) a wide variety of MALDI matrices or matrix-free sample supports are available to support the ionization of different biomolecules, 3) MALDI produces singly charged molecular ions, 4) TOF has the largest mass range in comparison to all other mass analyzers, 5) high mass resolution of 60,000 can be achieved with Spiral TOF [8] 6) measurement time for each sample can be as little as 1 second, 7) both data acquisition and spectral interpretation have been automated, 8) the same MALDI samples can be remeasured and/or archived, 9) imaging mass spectrometry (IMS), i.e. spatial distribution of different analytes, can be achieved by MALDI-TOF MS without switching to a different ion source [9], 10) tandem MALDI-TOF/TOF MS can provide relatively high colli-

${ }^{*}$ Corresponding author. sion-induced dissociation (CID) energy [10], and 11) accurate quantitation of biomolecule of interest can be achieved with suitable internal standard [11].

In general, quantitative MALDI-TOF MS can be divided into two different categories, namely relative and absolute quantitation. In the latter case, it is necessary to construct a calibration graph from measuring a series of standard dilutions in a sample matrix that is comparable to the samples. Equally important, in order to achieve a high level of accuracy on the quantitation, it is also necessary to determine the linearity (R-squared value) and the linear dynamic range in a calibration graph as well as the reproducibility of the measurements. Due to the heterogeneous morphology in MALDI sample-matrix cocrystals, one of the challenges in quantitative MALDITOF MS is the variation of signal intensity from repeated measurements of the same sample [12]. To overcome the variation of signal intensity, the most commonly used method is to add a fixed amount of an internal standard to each standard dilution [13-17]. Theoretically, with the same concentration of internal standard in each standard dilution, the internal standard signals should be constant. In this study, similar to the ion suppression that has been reported earlier, the signals of fixed amount of internal 
standard were significantly lowered over the linear dynamic range for quantitation. An attempt to experimentally identify the cause of reducing the internal standard signals was systematically carried out. Although the reduction of internal standard signals does not interfere with the quantitation of target analyte in the sample of interest, it represents a limitation on using MALDI-TOF MS to monitor the changes in concentration of two different compounds within a chemical or biological system.

\section{Experimental}

HPLC purified oligonucleotides (oligo) were ordered from Integrated DNA Technologies (Coralville, IA). A 17 mer oligo (5'-CCA TCC ACT ACA ACT AC-3') was used as a DNA standard and a 19 mer oligo (5' $-\mathrm{CCA}$ TCC ACT ACA ACT ACA T-3') was used as an internal standard, unless otherwise stated. 3-hydroxypicolinic acid (3-HPA) MALDI matrix was purchased from SigmaAldrich (St Louis, MO). Ammonium citrate dibasic $\geq$ 99.0\% was purchased from Fluka (Buchs, Switzerland). HPLC grade acetonitrile and methanol were obtained from Fisher Scientific (Pittsburgh, PA). Non-sterile 0.22 $\mu \mathrm{m}$ low protein binding Durapore (PVDF) syringe driven filter units $(25 \mathrm{~mm})$ were purchased from Millipore Corp. (Bedford, MA).

\section{Sample Preparation}

The 3-HPA matrix solution was prepared by dissolving $35.0 \mathrm{mg}$ of 3 -HPA and $7.1 \mathrm{mg}$ of ammonium citrate dibasic in $1.0 \mathrm{~mL}$ of $10 \%$ acetonitrile [18]. After vortexing the matrix solutions for $\sim 1 \mathrm{~min}$, any undissolved particles were removed by a $0.22 \mu \mathrm{m}$ filter. The matrix solution was made monthly and stored at $-20^{\circ} \mathrm{C}$. Both oligos were reconstituted to $250 \mu \mathrm{M}$ with autoclaved deionized water, and stored at $-20^{\circ} \mathrm{C}$. Prior to the MALDI-TOF MS measurements, both oligos were freshly diluted with autoclaved deionized water and mixed accordingly. The stainless steel MALDI sample plate was cleaned by rinsing the plate with deionized water and followed by methanol washing. $0.3 \mu \mathrm{L}$ of 3-HPA matrix solution was spotted on the MALDI sample plate, then allowed to air dry. $0.3 \mu \mathrm{L}$ of oligo mixture was then spotted over the dried matrix, and the mixture was allowed to air dry.

\section{MALDI-TOF MS Measurements}

All MALDI-TOF MS measurements were carried out by using a 4700 Proteomics Analyzer from Applied Biosystems (Framingham, MA). Each sample was measured by using the linear positive ion mode (linear middle mass positive acquisition method in the 4000 Series Explorer Version 3.0 software). The Nd:YAG laser intensity was set at 6000 arb. units (maximum intensity $=7900$ arb. units). The focus mass was the molecular mass of [DNA standard $+\mathrm{H}]^{+}$ion $(5044.3 \mathrm{Da})$. To achieve the highest mass resolution for the [DNA standard $+\mathrm{H}]^{+}$ion, $450 \mathrm{~ns}$ delay time was used. The accelerating voltage was +20.0 $\mathrm{kV}$ and grid voltage was $+18.2 \mathrm{kV}$. The instrument was equipped with a $200 \mathrm{~Hz}$ digitizer. The sampling bin size was $4 \mathrm{~ns}$ with an input bandwidth of $500 \mathrm{MHz}$, and a vertical full scale of $200 \mathrm{mV}$. The linear detector voltage was $+1.92 \mathrm{kV}$. The pressure inside the instrument was maintained at the level of $10^{-8}$ Torr. Each spectrum was automatically acquired with random edge-biased positioning of laser shots. The default width for local noise window in the linear middle mass positive processing method was $250 \mathrm{~m} / \mathrm{z}$, which defined the background noise from thebaseline within the specified mass window where a peak was detected in the mass spectrum. By using the Data Explorer Version 4.6 software, mass spectra were internally calibrated with the $[\text { DNA standard }+\mathrm{H}]^{+}$ion peak.

\section{Results and Discussion}

To achieve absolute quantitation of a specific analyte by using MALDI-TOF MS, it is necessary to perform a calibration with a series of pure standard dilutions. For the purpose of minimizing any possible errors in sample preparation and MALDI-TOF MS measurements, each standard dilution is usually measured at least three or four times, and the average signals are used to perform the calibration. It is known that one of the drawbacks on quantitative MALDI-TOF MS is the variation of signal intensity when the same sample is repeatedly measured. There are different experimental approaches that can be used to improve the reproducibility of MALDI-TOF MS measurements [19-21]. For performing calibration experiments, a common approach to overcome the variation of signal intensity is to add fixed amount of an internal standard to each standard dilution. By plotting the concentration of pure standard against the ratio of signals between pure standard and internal standard, linear calibration graphs can be constructed [13-17]. In this study, for the purpose of measuring DNA samples, a pure DNA oligonucleotide (17 mer) was used as a pure standard for calibration. To ensure the internal standard has similar physical and chemical properties as the pure DNA standard for performing the calibration, the internal standard used in this study was also a DNA oligonucleotide which had the same DNA sequence as the pure DNA standard, except two extra 2'-deoxynucleotides were added to its 3 ' end. In the calibration experiment, the final concentration of internal standard in each dilution of pure DNA standard was $1.00 \mu \mathrm{M}$. To minimize the effects of signal variation, each DNA mixture was measured twelve times. By using the average peak areas, a calibration graph was constructed as shown in Figure 1(a). The calibration graph has a linear dynamic range from $0.20 \mu \mathrm{M}$ to 12.5 
$\mu \mathrm{M}$ of DNA standard with an R-squared value of 0.997 . At the limit of quantitation, the average signal-to-noise ratio of $0.20 \mu \mathrm{M}$ DNA standard was equal to 3.73. According to the results in Figure 1(a), when the concentration of DNA standard and internal standard are equal to each other at $1.00 \mu \mathrm{M}$, the ratio of their corresponding peak areas is very much close to one. This indicates the incorporation of both DNA oligos in the MALDI matrix-sample co-crystals, and their efficiencies to be desorbed/ionized by the MALDI process are very similar to each other. In theory, the signals of fixed amount of internal standard should remain at the same level within the linear dynamic range of quantitation. However, when the signals of DNA standard and internal standard are separately plotted against the concentration of DNA standard as shown in Figure 1(b), the signals of internal standard
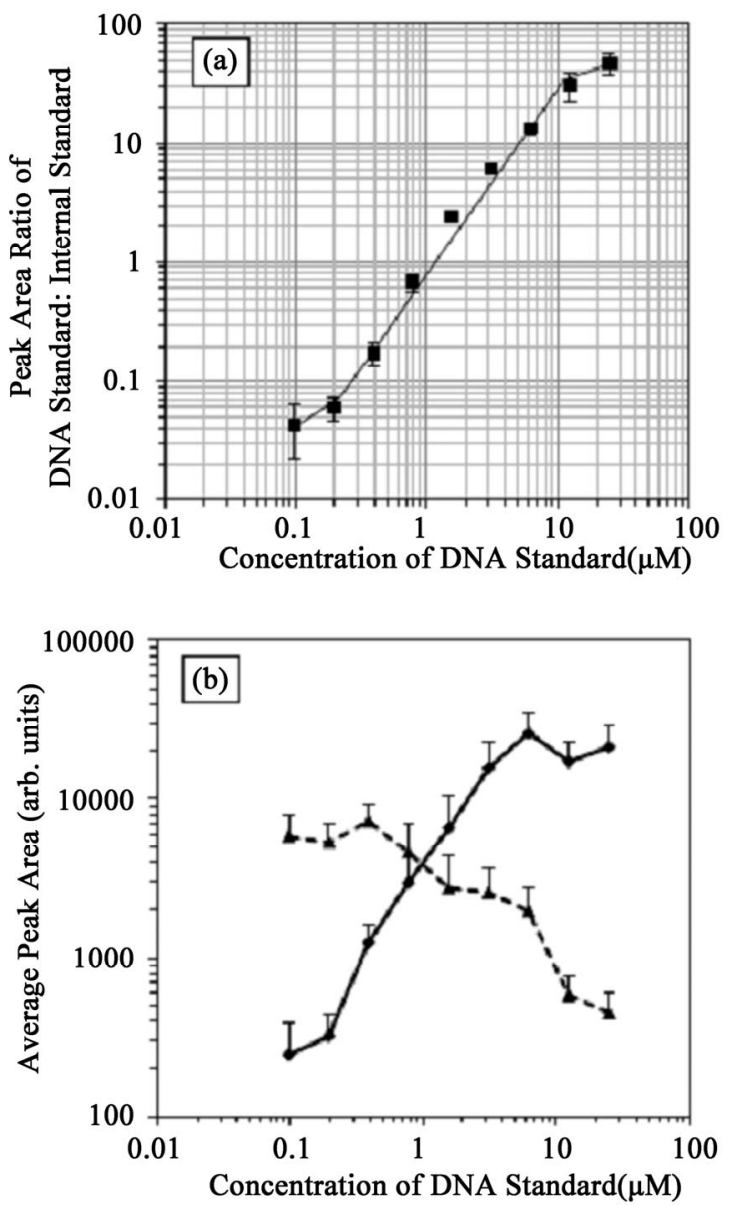

Figure 1. (a) Calibration graph for pure DNA standard. The pure DNA standard concentration $(0.10-25.0 \mu \mathrm{M})$ is plotted against the average peak area ratio of pure DNA standard to internal standard; (b) Unexpected decrease on the internal standard signals. The signals of DNA standard (solid line) and internal standard (dotted line) are plotted separately against the concentration of pure DNA standard. The experimental data in (a) and (b) are the same. All error bars represent one standard deviation $(n=12)$. are unexpectedly decreased from its initial level while the signals of DNA standard continue to increase proportionally with the increasing concentration of DNA standard. Specifically, the peak area of internal standard has decreased $73 \%$ from the initial level when the concentration of DNA standard was increased to $6.25 \mu \mathrm{M}$. At 6.25 $\mu \mathrm{M}$ of DNA standard, the peak area of DNA standard starts to level off. Similar reduction of various internal standard signals were found in the literature when the signal intensities are carefully examined [22-24]. These included the quantitative MALDI-TOF MS measurements of different nucleic acids, proteins, and synthetic polymers, in which different MALDI matrices and instrumentation were used. Thus, the unexpected decrease on the internal standard signal is not limited to MALDITOF mass spectrometry of DNA.

In an attempt to identify the cause of signal reduction in this study, the roles of the two DNA oligos were exchanged. Thus, the shorter 17mer DNA oligo was used as internal standard instead of the longer 19 mer DNA oligo. Based on the principle of the time-of-flight mass analyzer, the molecular ions of the shorter internal standard should reach the ion detector earlier than the longer 19 mer DNA oligo. This would ensure the ion detector was not saturated when the internal standard was detected. From measuring a series of DNA oligo mixtures that contained same amount of shorter internal standard (1.00 $\mu \mathrm{M})$ and different amounts of longer 19 mer DNA oligo, the results showed that the signals of shorter internal standard were also decreased while the signals of longer 19 mer DNA oligo continued to increase with its increasing concentrations (data not shown). Similar to the results in Figure 1(b), the peak area of the shorter internal standard has decreased $67 \%$ from the initial level. Thus, the same reduction of internal standard signals was observed when the roles of the two DNA oligos used in this study were interchanged. The unexpected decrease on the internal standard signals within the linear dynamic range was reproducible even when a different DNA oligo was used as an internal standard (data not shown). Together with the results in Figure 1(b), the unexpected decrease on the internal standard signals was neither due to any defect on measuring ions at a specific $\mathrm{m} / \mathrm{z}$ ratio nor due to the saturation of the ion detector.

Another possible explanation for the reduction of internal standard signals would be the initial MALDI process. Previously, based on the interpretation of MALDITOF MS spectra alone, Hercules and his co-workers have identified a similar reduction of signals when protein mixtures were measured [22]. Each protein mixture contained two different components, and one of them was a protein internal standard at a fixed concentration. To explain the phenomenon, they speculated the MALDI matrix was saturated by relative high concentration of 
protein analyte in the mixture. They had proposed a threshold value for the molar ratio between analyte and MALDI matrix to be at least 1:3000. In this study, the lowest molar ratio of (DNA standard + internal standard) to 3-HPA matrix within the linear dynamic range was $\sim 1: 19,000$. Hence, according to the Hercules's suggestion, there should be sufficient 3-HPA matrix to support the MALDI process, i.e. desorption and ionization of DNA oligos without any detectable laser induced fragmentation of DNA oligos. As shown in Figure 2(a), no fragment ion originated from the internal standard was observed. Thus, the signals of internal standard could not be reduced as a result of laser-induced fragmentation.

At neutral $\mathrm{pH}$, the phosphate groups along the phosphate backbone of a DNA molecule are deprotonated. When DNA oligo is mixed with acidic 3-HPA matrix $(\mathrm{pH}=3)$, the negatively charged phosphate groups are re-protonated. This occurs before the DNA sample is measured by MALDI-TOF MS. According to Knochenmusss's proposed ionization mechanism for the MALDI process, multiple matrix molecules are excited by the UV laser irradiation [25]. When two excited matrix molecules are close to each other, energy is redistributed and concentrated on one matrix molecule. This results in the formation of a matrix ion. To generate a positively charged DNA molecular ion in this study, DNA is ionized by gaseous-phase proton transfer from matrix ions. Based on this ionization mechanism, the minimum molar ratio between a DNA oligo and 3-HPA is 1:2. As mentioned earlier, the lowest molar ratio of DNA oligo to 3 -HPA in this study was $\sim 1: 19,000$. The excess of 3-HPA matrix ions was experimentally detected as

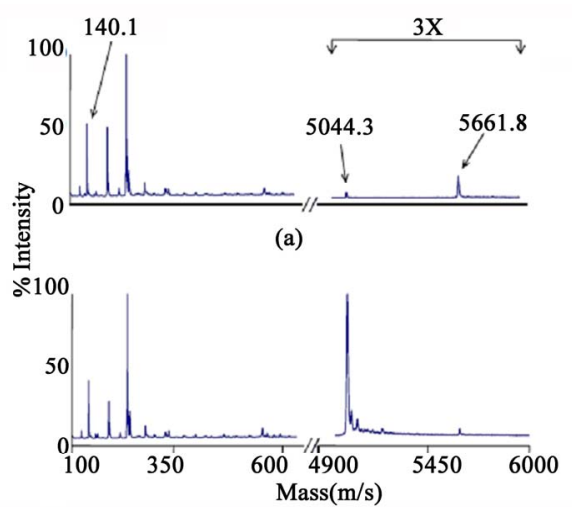

(b)

Figure 2. Excess 3-HPA MALDI matrix ions. Mass spectra of $0.20 \mu \mathrm{M}$ (a) and $12.5 \mu \mathrm{M}$ (b) of pure DNA standard (5044.3 Da) with the same concentration $(1.00 \mu \mathrm{M})$ of internal standard $(5661.8 \mathrm{Da})$. In both cases, the excess of $[3 \mathrm{HPA}+\mathrm{H}]^{+}$ion (140.1 Da) and its cluster ions (unlabeled) were detected in the low mass window. The signal intensity in the high mass window has been increased 3 times. Both spectra were acquired with the low mass gate being disabled, and the mass range was set between $100 \mathrm{Da}$ and $6000 \mathrm{Da}$. shown in Figure 2. When comparing the signal that correspond to $[3-\mathrm{HPA}+\mathrm{H}]^{+}$ion $(140.1 \mathrm{~m} / \mathrm{z})$ obtained from the lowest and the highest amount of DNA standard used in the calibration experiment, the signal intensities of [3-HPA $+\mathrm{H}]^{+}$ion are comparable (Figure 2). Thus, even at the highest DNA standard concentration, there were still excess MALDI matrix ions to support the ionization of DNA oligo molecules. In other words, the decrease on the internal standard signals could not be explained by the lack of MALDI matrix ion. With excess MALDI matrix ions and the same signal reduction was observed when the roles of the two DNA oligos were exchanged, the decrease on the internal standard signals could not be completely explained by the analyte suppression effects that have been reported in the literature [26].

In general, the yield of molecular ions from a MALDI sample could be increased by using higher laser intensity [27]. To balance between the yield of molecular ions and its possible laser-induced fragmentation, the optimal laser intensity for measuring the DNA standard was determined to be at 6000 arb. units in a separate study. With a fixed laser intensity, there should be a limitation on generating DNA molecular ions. In Figure 3, the concentration of pure DNA standard is plotted against the sum of signals obtained from both pure DNA standard and internal standard. The sum of signals continues to increase with the concentration of pure DNA standard, whereas the internal standard signal begins to decrease at $0.39 \mu \mathrm{M}$ of pure DNA standard (Figure 1(b)). The continuous increase in the sum of signals does comply with the earlier discussion that there were excess MALDI matrix ions to support the desorption/ionization of both internal standard and DNA standard within the linear dynamic range. Also, the continuous increase in the sum of signals

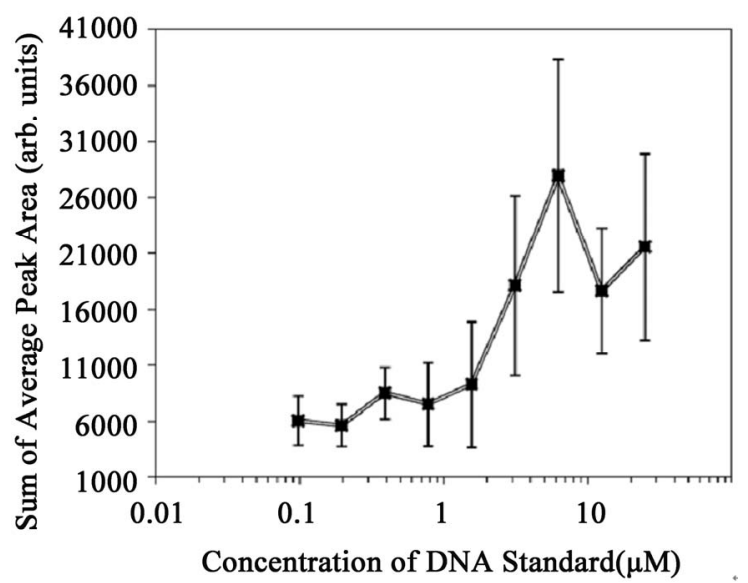

Figure 3. Capacity of MALDI ion source for producing molecular ions. The sum of signals obtained from both pure DNA standard and internal standard is plotted against the concentration of DNA standard. All error bars represent one standard deviation $(n=12)$. 
indicated the capacity of the ion source to produce molecular ions had not been exceeded when the internal standard signals began to decrease.

\section{Conclusion}

Despite several studies had used fixed amount of internal standard to achieve accurate quantitation of different analytes by using MALDI-TOF MS, this is the first time the extent of signal reduction from fixed amount of internal standard is reported. Based on the results of this study, the cause for the unexpected decrease on the internal standard signals could not be explained by the choice of internal standard, any defect associated with the desorption/ionization of the internal standard, and the detection of the internal standard ion. A possible explanation for the reduction of internal standard signals could be some of its molecular ions are discharged as a result of encountering ions with the opposite charge, and/or colliding with a conductive surface within the MALDI ion source [28]. Nevertheless, it is important to realize the unexpected signal reduction represents a limitation on using quantitative MALDI-TOF MS to monitor the changes on the concentrations of two different compounds in a chemical or biological system, especially when their corresponding signals were directly compared. This limitation, however, is not applicable when one of the two compounds of interests is at a constant level and the ratio of the two corresponding signals is used to monitor the changes on the other compound.

\section{Acknowledgements}

The authors wish to thank NadjaCech and Liam Duffy for helpful discussion. All financial support was received from UNCG. The MALDI-TOF/TOF instrument was purchased with a NSF Major Research Instrumentation Grant (Award \#0420292).

\section{REFERENCES}

[1] D. Fenyö and R. C. Beavis, "Informatics Development: Challenges and Solutions for MALDI Mass Spectrometry," Mass Spectrometry Reviews, Vol. 27, No. 1, 2008, pp. 1-19. doi:10.1002/mas.20152

[2] J. Ragoussis, G. P. Elvidge, K. Kaur and S. Colella, "Matrix-Assisted Laser Desorption/Ionisation, Time-of-Flight Mass Spectrometry in Genomics Research," PLoS Genetics, Vol. 2, 2006, p. e100.

doi:10.1371/journal.pgen.0020100

[3] S. Douthwaite and F. Kirpekar, "Identifying Modifications in RNA by MALDI Mass Spectrometry," Methods in Enzymology, Vol. 425, 2007, pp. 1-20. doi:10.1016/S0076-6879(07)25001-3

[4] J. Schiller, R. Suss, B. Fuchs, M. Muller, O. Zschornig and K. Arnold, "MALDI-TOF MS in Lipidomics," Fron- tiers in Bioscience, Vol. 12, No. 7, 2007, pp. 2568-2579. doi: $10.2741 / 2255$

[5] M. L. Reyzer and R. M. Caprioli, "MALDI-MS-Based Imaging of Small Molecules and Proteins in Tissues," Current Opinion in Chemical Biology, Vol. 11, No. 1, 2007, pp. 29-35. doi:10.1016/j.cbpa.2006.11.035

[6] D. J. Harvey, "Structural Determination of N-Linked Glycans by Matrix-Assisted Laser Desorption/Ionization and Electrospray Ionization Mass Spectrometry," Proteomics, Vol. 5, No. 7, 2005, pp. 1774-1786.

doi:10.1002/pmic.200401248

[7] G. Bolbach, "Matrix-Assisted Laser Desorption/Ionization Analysis of Non-Covalent Complexes: Fundamentals and Applications," Current Pharmaceutical Design, Vol. 11, No. 20, 2005, pp. 2535-2557. doi: $10.2174 / 1381612054546923$

[8] T. Satoh, T. Sato and J. Tamura, "Development of a High-Performance MALDI-TOF Mass Spectrometer Utilizing a Spiral Ion Trajectory," Journal of the American Society for Mass Spectrometry, Vol. 18, No. 7, 2007, pp. 1318-1323. doi:10.1016/i.jasms.2007.04.010

[9] A. Chacon, I. Zagol-Ikapitte, V. Amarnath, M. L. Reyzer, J. A. Oates, R. M. Caprioli and O. Boutaud, "On-Tissue Chemical Derivatization of 3-Methoxysalicylamine for MALDI-Imaging Mass Spectrometry," Journal of Mass Spectrometry, Vol. 46, No. 8, 2011, pp. 840-846. doi:10.1002/jms.1958

[10] C. A. Barnes and N. H. L. Chiu, "Accurate Characterization of Carcinogenic DNA Adducts Using MALDI Tandem Time-of-Flight Mass Spectrometry," International Journal of Mass Spectrometry, Vol. 279, No. 2-3, 2009, pp. 170-175. doi:10.1016/j.ijms.2008.10.006

[11] W. Yang and N. Chiu, "Comparison of Accuracy on DNA Quantitation Determined by MALDI-TOF Mass Spectrometry and UV Spectrometry," Spectroscopy Letters, Vol. 43, No. 7-8, 2010, pp. 602-608. doi:10.1080/00387010.2010.510766

[12] R. W. Garden and J. V. Sweedler, "Heterogeneity within MALDI Samples as Revealed by Mass Spectrometric Imaging," Analytical Chemistry, Vol. 72, No. 1, 2000, pp. 30-36. doi:10.1021/ac9908997

[13] L. K. Zhang and M. L. Gross, "Matrix-Assisted Laser Desorption/Ionization Mass Spectrometry Methods for Oligodeoxynucleotides: Improvements in Matrix, Detection Limits, Quantification, and Sequencing," Journal of the American Society for Mass Spectrometry, Vol. 11, No. 10, 2000, pp. 854-865. doi:10.1016/S1044-0305(00)00161-6

[14] W. T. Berggren, T. Takova, M. C. Olson, P. S. Eis, R. W. Kwiatkowski and L. M. Smith, "Multiplexed Gene Expression Analysis Using the Invader RNA Assay with MALDI-TOF Mass Spectrometry Detection," Analytical Chemistry, Vol. 74, No. 8, 2002, pp. 1745-1750. doi:10.1021/ac011167t

[15] K. Tang, S. L. Allman, R. B. Jones and C. H. Chen, "Quantitative Analysis of Biopolymers by Matrix-Assisted Laser Desorption," Analytical Chemistry, Vol. 65, No. 15, 1993, pp. 2164-2166. doi:10.1021/ac00063a041

[16] D. Sarracino and C. Richert, "Quantitative MALDI-TOF 
MS of Oligonucleotides and a Nuclease Assay," Bioorganic \& Medicinal Chemistry Letters, Vol. 6, No. 21, 1996, p. 3057. doi:10.1016/0960-894X(96)00465-9

[17] B. A. Bruenner, T.-T. Yip and T. W. Hutchens, "Quantitative Analysis of Oligonucleotides by Matrix-Assisted Laser Desorption/Ionization Mass Spectrometry," Rapid Communications in Mass Spectrometry, Vol. 10, No. 14, 1996, pp. 1797-1801. doi:10.1002/(SICI)1097-0231(199611)10:14<1797::AID$\underline{\mathrm{RCM} 754>3.0 . \mathrm{CO} ; 2-5}$

[18] K. Tang, S. L. Allman and C. H. Chen, "Matrix-Assisted Laser Desorption Ionization of Oligonucleotides with Various Matrices," Rapid Communications in Mass Spectrometry, Vol. 7, No. 10, 1993, pp. 943-948. doi: $10.1002 / \mathrm{rcm} .1290071016$

[19] J. M. Koomen, W. K. Russell, J. M. Hettick and D. H. Russell, "Improvement of Resolution, Mass Accuracy, and Reproducibility in Reflected Mode DE-MALDI-TOF Analysis of DNA Using Fast Evaporation-Overlayer Sample Preparations," Analytical Chemistry, Vol. 72, No. 16, 2000, pp. 3860-3866. doi:10.1021/ac0001941

[20] A. Honda, H. Sonobe, A. Ogata and K. Suzuki, "Improved Method of the MALDI-TOF Analysis of DNA with Nanodot Sample Target Plate," Chemical Communications, Vol. 42, 2005, pp. 5340-5342. doi:10.1039/b507065a

[21] L. J. Dekker, J. C. Dalebout, I. Siccama, G. Jenster, P. A. Sillevis Smitt and T. M. Luider, "A New Method to Analyze Matrix-Assisted Laser Desorption/Ionization Timeof-Flight Peptide Profiling Mass Spectra," Rapid Communications in Mass Spectrometry, Vol. 19, No. 7, 2005, pp. 865-870. doi:10.1002/rcm.1864

[22] A. I. Gusev, W. R. Wilkinson, A. Proctor and D. M. Hercules, "Direct Quantitative Analysis of Peptides Using
Matrix Assisted Laser Desorption Ionization," Fresenius' Journal of Analytical Chemistry, Vol. 354, No. 4, 1996, pp. 455-463. doi:10.1007/s0021663540455

[23] B. A. Garcia, P. J. Heaney and K. Tang, "Improvement of the MALDI-TOF Analysis of DNA with Thin-Layer Matrix Preparation," Analytical Chemistry, Vol. 74, No. 9, 2002, pp. 2083-2091. doi:10.1021/ac011089+

[24] H. Chen and M. He, "Quantitation of Synthetic Polymers Using an Internal Standard by Matrix-Assisted Laser Desorption/Ionization Time-of-Flight Mass Spectrometry," Journal of the American Society for Mass Spectrometry, Vol. 16, No. 1, 2005, pp. 100-106. doi:10.1016/j.jasms.2004.09.024

[25] R. Knochenmuss, "Photoionization Pathways and Free Electrons in UV-MALDI," Analytical Chemistry, Vol. 76, No. 11, 2004, pp. 3179-3184. doi:10.1021/ac035501s

[26] Y. Kong, Y. Zhu and J. Y. Zhang, "Ionization Mechanism of Oligonucleotides in Matrix-Assisted Laser Desorption/ Ionization Time-of-Flight Mass Spectrometry," Rapid Communications in Mass Spectrometry, Vol. 15, No. 1, 2001, pp. 57-64.

doi:10.1002/1097-0231(20010115)15:1<57::AID-RCM19 2>3.0.CO; $2-7$

[27] R. Knochenmuss, "A Quantitative Model of Ultraviolet Matrix-Assisted Laser Desorption/Ionization Including Analyte Ion Generation," Analytical Chemistry, Vol. 75, No. 10, 2003, pp. 2199-2207. doi:10.1021/ac034032r

[28] G. Westmacott, W. Ens, F. Hillenkamp, K. Dreisewerd and M. Schurenberg, "The Influence of Laser Fluence on Ion Yield in Matrix-Assisted Laser Desorption Ionization Mass Spectrometry," International Journal of Mass Spectrometry, Vol. 221, No. 1, 2002, pp. 67-81. doi:10.1016/S1387-3806(02)00898-9 